\title{
ANALOGUES OF ARTIN'S CONJECTURE ${ }^{1}$
}

\author{
BY LARRY JOEL GOLDSTEIN
}

Communicated by W. Feit, November 17, 1967

Artin's celebrated conjecture on primitive roots (Artin [1, p. viii], Hasse [2], Hooley [3]) suggests the following

Conjecture. Let $S^{\prime}$ be a set of rational primes. For each $q \in S$, let $L_{q}$ be an algebraic number field of degree $n(q)$. For every square-free in teger $k$, divisible only by primes of $S$, define $L_{k}$ to be the composite of all $L_{q}, q \mid k$, and denote $n(k)=\operatorname{deg}\left(L_{k} / Q\right)$. Assume that $\sum_{k} 1 / n(k)$ converges, where the sum is over those $k$ for which $L_{k}$ is defined. Then the natural density of the set $P$ of all primes $p$ which do not split completely in each $L_{q}$ exists and has the value $\sum_{k} \mu(k) / n(k)$, where $\mu$ is the Möbius function and the term $k=1$ has been included with $n(1)=1$.

If $S=\{$ all rational primes $\}, L_{q}=Q\left(\zeta_{q}, a^{1 / q}\right), a \in Z, \zeta_{q}=$ a primitive $q$ th root of 1 , then the conjecture is equivalent to Artin's conjecture. If $S$ is a finite set, then the conjecture is easily verifiable using the prime ideal theorem. For $S=\{$ all rational primes $\}, L_{q}=Q\left(\zeta_{q^{r}}\right)$, the conjecture has been proved by Knobloch [4] (for $r=2$ and only for Dirichlet densities) and by Mirsky [5].

We have proved the following theorems, whose proofs will appear elsewhere.

THEOREM 1. Let there exist a finite set $S_{0} \subset S$ such that $L_{q} \supset Q\left(\zeta_{q}^{2}\right)$ for $q \in S-S_{0}$, and $L_{q} / Q$ is normal for all $q \in S$. Then the conjecture is true.

THEOREM 2. Suppose that for each finite subset $S_{0} \subset S$ there exists a family of algebraic number fields $\left\{L_{q}^{\prime}\right\}_{\alpha \in S}$ such that

(1) $L_{q}=L_{q}^{\prime}$ for $q \in S_{0}$,

(2) $L_{q}^{\prime} \subset L_{q}$ for all $q \in S$,

(3) $L_{q}^{\prime} \neq Q$ for all $q \in S$,

(4) the conjecture is true for $\left\{L_{q}^{\prime}\right\}_{q \in S}$.

Then the conjecture is true for $\left\{L_{q}\right\}_{Q \in S}$.

Theorem 3. If the density $d(P)$ of $P$ exists, then

$$
d(P) \leqq \sum_{k} \mu(k) / n(k) .
$$

${ }^{1}$ Research partially supported by Air Force Office of Scientific Research Grant No. SAR/F-44620-67. 
Theorem 1 is the main result. Theorems 2 and 3 are elementary in character. The proof of Theorem 1 is divided into two parts: First, it is shown that one may compute the number of primes $p \leqq x$ in $P$ by computing the number of primes $p \leqq x$ which do not split completely in $L_{q}$ for all "sufficiently small $q$ " where the upper bound for $q$ is a function of $x$. Computing this latter quantity is reduced to computing the number of prime ideals of $L_{k}$ which have norm $\leqq x$, for all "sufficiently small $k$." The prime ideal theorem asserts that this latter quantity is asymptotically equal to $x / \log x$. But the error term will, in general, depend on $L_{k}$. The second part of the proof consists in showing that by restricting $k$ to be "sufficiently small" one can choose the error term to be independent of $k$. This result constitutes a generalization of the uniform prime number theorem of Siegel and Walfisz (Prachar [6, p. 144]) for primes in arithmetic progressions. In fact, we can prove our theorem in a very general setting, which, although not required for the proofs of Theorems 1-3, seems interesting for its own sake.

Let $K$ be a normal algebraic number field of degree $n$ and discriminant $d$. Let $\alpha \rightarrow \alpha^{(j)}(1 \leqq j \leqq n)$ be the embeddings of $K$ in the complex numbers $C$, ordered so that the first $r_{1}$ are real and the $j$ th and $\left(j+r_{2}\right)$ th $\left(r_{1}+1 \leqq j \leqq r_{1}+r_{2}\right)$ constitute a pair of complex-conjugate embeddings. Let

$$
\begin{aligned}
& n_{j}=1, \quad 1 \leqq j \leqq r_{1} \\
&=2, \quad r \\
& r_{1}+1 \leqq r_{1}+r_{2} .
\end{aligned}
$$

For $\alpha \in K^{*}=K-\{0\}$, let $\alpha \equiv 1\left(\bmod ^{*} \mathfrak{a}\right)$ mean that $\alpha$ is multiplicatively congruent to 1 modulo the $K$-ideal a. For $\alpha \in K^{*}$, denote by $(\alpha)$ the $K$-ideal generated by $\alpha$. Let $\chi$ be a grossencharacter of $K$ having conductor $\mathrm{f}$. For $\alpha \equiv 1\left(\bmod ^{*} \mathrm{f}\right)$, let

$$
\chi((\alpha))=\prod_{j=1}^{r_{1}+r_{2}}\left(\frac{\alpha^{(j)}}{\left|\alpha^{(j)}\right|}\right)^{m_{j}}\left|\alpha^{(j)}\right|{ }^{i n j \phi_{j}}
$$

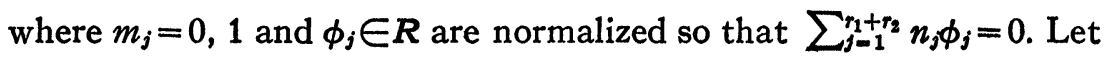

$$
\pi(x, K, \chi)=\sum_{N p_{\leq} x ;(p, f)-1} \chi(p)
$$

where the sum is over primes $\mathfrak{p}$ of $K$. For $A>0$, define $B(A)=\{x$ a grossencharacter of $\left.K|| \phi_{j} \mid \leqq A, 1 \leqq j \leqq r_{1}+r_{2}\right\}$. Then we have the following generalization of the Siegel-Walfisz theorem:

Theorem 4. Let $A>0, \epsilon>0$ be given. Then there exists a positive constant $c=c(A, \epsilon)$, not depending on $K, n, d$, or $\chi$ such that for $x \in B(A)$, 


$$
\pi(x, K, \chi)=E(\chi) \text { li } x+O\left(D x \log ^{2} x \exp \left\{-\operatorname{cn}(\log x)^{1 / 2} / D\right\}\right), x \rightarrow \infty
$$

where the 0-term constant does not depend on $K, \chi, n$ or $d$ and

$$
\begin{aligned}
E(\chi) & =0, \quad \chi \neq \text { the trivial grossencharacter } \\
& =1, \quad \chi=\text { the trivial grossencharacter, } \\
\operatorname{li} x & =\int_{2}^{x} \frac{d y}{\log y}, \\
D & =n^{4}[|d| N(\mathfrak{f})]^{e} c^{-n} .
\end{aligned}
$$

\section{BIBLIOGRAPHY}

1. E. Artin, The collected works of Emil Artin, Addison-Wesley, Reading, Mass., 1965.

2. H. Hasse, Über die artinsche Vermutung und verwandte Dichtefragen, Ann. Acad. Sci. Fenn., Ser. A. I 116 (1952).

3. C. Hooley, On Artin's conjecture, J. Reine Angew. Math. 225 (1967), 209-220.

4. W. Knobloch, Über Primzahlreihen nebst Anwendung auf ein elementares Dichteproblem, Abh. Math. Sem. Univ. Hamburg 19 (1953), 1-13.

5. L. Mirsky, The number of representations of an integer as the sum of a prime and $a$ k-free integer, Amer. Math. Monthly 56 (1949), 17-19.

6. K. Prachar, Primzahlverteilung, Die Grundlehren der mathematischen Wissenschaften, Band 91, Springer-Verlag, Berlin, 1957.

YaLE UNIVERSITY 